\title{
Prevalence of pressure ulcers, risk factors and use of pressure- relieving mattresses in ICU
} patients

Jan Weststrate, RN, CCRN, MSc, Clinical Researcher, Department of General Surgery, University Hospital, Rotterdam, The Netherlands ( $\bullet$ )

Freerk Heule, MD, PhD, Department of Dermatology, University Hospital, Rotterdam, The Netherlands

JTM Weststrate, University Hospital Rotterdam, Room Z-638, PO Box 2040, 3000 CA, Rotterdam, The Netherlands.

e-mail: weststrate@aziv.azr.nl

\section{INTRODUCTION}

Preventing the development of a pressure ulcer is an important aspect of the care provided by nurses and doctors. As well as being a very painful and uncomfortable complication, pressure ulcers also affect nursing care as they increase the nursing workload per patient by $50 \%$ (Barrett, 1990). Furthermore, it is a very costly problem. In the Netherlands alone, it is estimated that approximately 700 million Euros are spent each year on the prevention and treatment of pressure ulcers (Haalboom, 1992). Another study has calculated the cost of treating a pressure ulcer per patient as between 5,500 and 45,000 Euros (Inman et al., 1993). Although prevention is 'treating' something unseen, prevention is still the best and cheapest option compared to the cost of active treatment of a pressure ulcer (Xakellis and Frantz, 1996).

In general, there are three patient groups that run a particularly high risk in developing a pressure ulcer. Besides patients with spinal cord injuries or geriatric patients, patients in an intensive care unit (ICU) are particularly prone to developing pressure ulcers. Jiricka et al. (1995) found that $25 \%$ of patients developed a pressure ulcer greater than stage II severity during their stay on the ICU. If pressure ulcers of stage I severity were included, the percentage of patients who developed a pressure ulcer increased to $56 \%$ (Jiricka et al., 1995). In a more recent study, Fife et al. (2001) found that the incidence of pressure ulcers (stage II or higher severity) was $12.4 \%$ in ICU patients. In another study, the incidence of pressure ulcers (stage II or higher) was found to be $7.9 \%$ among 594 surgical ICU patients (Weststrate et al., 1998).

These results demonstrate the large differences in incidence data between different ICUs. This might be caused by the fact that different groups of patients were included in these studies, but may also be due to the differences in care being given. This last issue is difficult to discuss among nurses, because in the past the development of pressure ulcers has often been associated with poor basic patient care (Dealey, 1991). Nowadays, how- ever, the problem of pressure ulcers in most institutions is seen as a multifactorial, multidisciplinary problem that needs a multidisciplinary approach to be solved. The influence of the European Pressure Ulcer Advisory Panel (EPUAP) may have contributed to this change in perception, as this organisation includes doctors, nurses, technicians and scientists.

\section{Pressure ulcer risk assessment scales}

There are a variety of pressure ulcer risk assessment scales, which are used in ICU patients to quantify the level of risk (low, medium, high and extremely high). These can help ICU nurses to identify early on those patients needing preventive intervention to stop the development or worsening of a pressure ulcer. Different assessment scales have been used by different investigators. For example, the Braden risk assessment scale was used in the study by Fife et al. (2001), Carlson et al. (1999) and Jiricka et al. (1995), while Weststrate et al. (1998) used the Waterlow risk assessment scale, and Jackson (1999) used the Jackson/Cubbin pressure area risk calculator. It is important to realise that these assessment scales are not predictive instruments, as they do not predict the development of a pressure ulcer, irrespective of what the intensive care nurse does to try to prevent it. Instead, these scales provide a clinical indication of the risk a patient has of developing a pressure ulcer if no preventive interventions are taken (Waterlow, 1995).

\section{Risk factors}

Several studies have identified risk factors for pressure ulcers which are particularly associated with ICU patients. Batson et al. (1993) identified four highly significant, critical care-related factors:

noradrenaline infusions

- adrenaline infusions 
- restrictive movement, due to traction, post-operative pain, intra-aortic balloon pump and haemofiltration

- patients who were too unstable to turn (Batson et al., 1993).

Another study found that extracorporeal circulation and time on the operating table were significant factors (Kemp et al., 1990), while another found sensory perception of the patient was a critical factor (Carlson et al., 1999). From three national prevalence studies done in the Netherlands, Bours et al. (2001) found that the most significant risk factors were: age; day since admission; malnutrition; and the three Braden subscales, moisture, sensory perception and mobility. Thus, specific risk factors can be identified, though different studies emphasise different factors.

\section{Pressure-relieving mattresses}

In the area of critical care, the use of pressure-relieving mattresses is an essential component in the prevention of pressure ulcers. Patients in ICU can develop a pressure ulcer within hours due to the above mentioned risk factors. The availability and readiness of special support surfaces that relieve pressure and therefore stimulate skin perfusion are essential. Having to wait for another 12-24 hours for a special mattress can be detrimental to the skin of the intensive care patient (Carlson et al., 1999).

\section{Prevalence of pressure ulcers}

From a European perspective, O'Dea (1995) investigated the prevalence of pressure ulcers in four countries (Netherlands, Italy, Germany and the UK); prevalence ranged from $7-18 \%$. So far, no study has investigated whether there is a difference in pressure ulcer prevalence between ICUs in different countries. As Europe becomes more integrated and European nations work together more closely, knowledge of pressure ulcer prevention strategies used by the various ICUs in Europe can be gathered to identify more and less successful strategies. This study investigates the prevalence of pressure ulcers in intensive care patients in four European countries and looked at factors that could possibly influence the results.

\section{MATERIAL AND METHODS}

A questionnaire, specially developed for this study, was distributed among ICUs in Denmark, Germany, Italy and the Netherlands. Part one of the questionnaire asked questions about the organisation of the ICU in relation to pressure ulcer prevention (Table 1). Part two asked questions about the risk, existence and prevention strategies for each patient in the ICU (Table 1).

The nurse managers of the different ICUs were approached by national representatives of KCI Medical (Houten, The Netherlands), and asked to participate in the study. If participation was agreed, the KCI representative explained the questionnaire to the nurse manager and the nursing team. The management of the ICU was asked to choose a day in the following week, on which to complete a questionnaire for each patient present that day on the unit at $8.00 \mathrm{am}$. Part one of the questionnaire was filled in by the nurse manager and part two by the ICU nurse looking after the patient at the time of assessment. When the data had been collected, it was checked and signed by the nurse manager for authenticity and sent to the Dutch office of KCI Medical, where data was entered into a Microsoft Access database. After all the data had been entered, the data entry was checked for accuracy and the data file with the original data was sent to the investigators where it was analysed using the statistical software programme SPSS 9.0.

\section{Table 1. Study questionnaire provided to ICUs}

Part 1: Questions answered by the nurse manager about organisational issues in relation to prevention of pressure ulcers on the ICU

Presence of a specialist nurse on the unit or in the hospital to advise regarding assessment of the risk of pressure ulcer development and its prevention

- Name of pressure ulcer risk assessment scale

- Frequency of pressure ulcer risk assessment

- Existence of a hospital pressure ulcer prevention and treatment protocol on the unit

Part 2: Patient observations answered by the ICU nurse in relation to pressure ulcer prevention

- Demographic data

Risk of pressure ulcer development according to the pressure ulcer risk scale used (high, medium or low)

Surgery in the previous 24 hours or more than 24 hours ago

- Usage of vasoactive medication in the previous 24 hours

- Mobility (immobile, changes position sometimes, changes position frequently)

- Activity (inactive, on chair, active)

- Sensitivity (no reaction to stimuli, limited reaction, normal reaction)

- Presence of incontinence and oedema

- Presence of pressure ulcers at sacrum and heel (grade $\geq 2$ )

- Type of nursing interventions taken to prevent pressure ulcer development

Type of surface upon which the patient is lying

\section{RESULTS}

\section{Participating critical care units}

A total of 44 ICUs were evaluated, with a total of 299 patients, during the period of March 2000 to June 2000. Table 2 shows the distribution of the ICUs in the participating countries. The majority were general ICUs $(n=29)$ and coronary care units $(n=8)$. The remaining ICUs were medical $(n=1)$, neurological $(n=2)$, surgical $(n=3)$ and cardiothoracic $(n=1)$. The average number of beds on each unit was 8.7 (range between 3 and 18). Of all the ICUs, $71 \%$ used a hospital pressure ulcer prevention and treatment protocol and $61 \%$ had a nurse specialised in the prevention and treatment of pressure ulcers in the hospital. $41 \%$ of the units actually had a pressure ulcer specialist nurse working on the unit.

In relation to the use of a pressure ulcer risk assessment scale, the Norton risk assessment scale was used on most units $(43.2 \%)$, the Waterlow scale in $6.8 \%$, the Braden in $4.5 \%$ and the Dutch CBO scale in $4.5 \%$. On $27.3 \%$ of the units, the nurses used their own clinical judgement instead of an existing scale to evaluate the patients' risk of developing a pressure ulcer. On $9.1 \%$ of the units, the nurses used their local scale, while $4.5 \%$ of the units used another scale, the name of which was not mentioned. $25 \%$ of the units used their risk assessment scale during every shift and $36 \%$ did this daily. A risk assessment scale was used by $34 \%$ of the units once a week, while $5 \%$ of units never carried out any form of risk assessment.

\section{Patients}

A total of 299 patients participated in the study. Table 2 gives an

\begin{tabular}{|c|c|c|c|}
\hline Country & $\begin{array}{l}\text { No. of participating } \\
\text { critical care units }\end{array}$ & No. of patients & $\begin{array}{l}\text { No. of patients with } \\
\text { a pressure ulcer }\end{array}$ \\
\hline Denmark & 3 & 24 & 1 \\
\hline Italy & 25 & 150 & 21 \\
\hline Germany & 11 & 99 & 49 \\
\hline Netherlands & 5 & 26 & 10 \\
\hline Total & 44 & 299 & 81 \\
\hline
\end{tabular}


overview of the number of patients with a pressure ulcer, while Table 3 provides the demographic data for all patients in the study. Of the total number of patients, $27 \%$ had a pressure ulcer stage II or greater. The prevalence of presssure sores varied between countries; in Denmark, it was $4 \%$, in Italy $14 \%$, in Germany $49 \%$, and in The Netherlands 38\%.

At the moment of observation, intensive care nurses were asked to classify their patient as having a high, medium or low risk in developing a pressure ulcer, according to the risk assessment scale used on their unit. Overall, 150 patients were identified as being at high risk of developing a pressure ulcer, of whom $31 \%$ already had a pressure ulcer. Of patients with a medium risk of developing a pressure ulcer $(\mathrm{n}=88), 27 \%$ already had a pressure ulcer. Of patients at low risk of developing a pressure ulcer $(n=29), 3 \%$ had a pressure ulcer. In Germany, among high-risk patients with no pressure ulcers, $63 \%$ were being given preventive treatment with a special mattress; in the other countries in the study, this figure was $85 \%$. Table 4 shows the risk level for pressure ulcers by country.

\section{Risk factors}

The study observed the following risk factors for developing a pressure ulcer:

mobility
activity
sensitivity
vasoactive medication
incontinence
oedema
surgery less than 24 hours ago
surgery more than 24 hours ago.

Table 5 shows the frequency that these factors were present in all patients and specifically in those the patients who had developed a pressure ulcer.

\section{Support mattress}

According to the risk level of developing a pressure ulcer, results indicate that $80 \%$ of high-risk patients were using a special support mattress, $64 \%$ of medium-risk patients and $54 \%$ of low-risk patients. Table 6 shows the frequency of choice for each of the types of commonly used support mattresses, for patients with and without a pressure ulcer.

\begin{tabular}{lcc}
\multicolumn{3}{l}{ Table 3. Demographic data of participating patients (SD) } \\
\hline & Female & Male \\
\hline Age (years) & $62( \pm 20)$ & $59( \pm 18)$ \\
Height $(\mathrm{m})$ & $1.63( \pm 0.13)$ & $1.74( \pm 0.13)$ \\
Weight $(\mathrm{kg})$ & $67( \pm 22)$ & $76( \pm 18)$ \\
Days in ICU & $11( \pm 23)$ & $17( \pm 30)$
\end{tabular}

\begin{tabular}{|c|c|c|c|c|c|c|c|c|}
\hline \multirow[b]{2}{*}{ Level of Risk } & \multicolumn{2}{|c|}{ Denmark } & \multicolumn{2}{|c|}{ Germany } & \multicolumn{2}{|l|}{ Italy } & \multicolumn{2}{|c|}{ Netherlands } \\
\hline & $\mathrm{n}$ & n-pu(\%) & $\mathrm{n}$ & n-pu (\%) & $\mathrm{n}$ & n-pu(\%) & $\mathrm{n}$ & $\mathrm{n}-\mathrm{pu}(\%)$ \\
\hline High & 16 & $1(6)$ & 37 & $21(57)$ & 79 & 15 (19) & 18 & $10(56)$ \\
\hline Medium & 6 & 0 & 43 & $21(49)$ & 34 & $3(9)$ & 5 & 0 \\
\hline Low & 1 & 0 & 6 & $1(17)$ & 19 & 0 & 3 & 0 \\
\hline Missing data & 1 & 0 & 13 & $6(46)$ & 18 & $3(17)$ & 0 & 0 \\
\hline Total of patients & 24 & & 99 & & 150 & & 26 & \\
\hline
\end{tabular}

Table 5. Number of patients, without a pressure ulcer, but with a specific risk factor at the time of assessment ( $\mathrm{n}$-total) compared to the number of patients with a pressure ulcer in whom a specific risk factor is present (n-pu)

\begin{tabular}{|c|c|c|c|c|c|c|c|c|}
\hline & \multicolumn{2}{|c|}{ Denmark } & \multicolumn{2}{|c|}{ Germany } & \multicolumn{2}{|l|}{ Italy } & \multicolumn{2}{|c|}{ Netherlands } \\
\hline Risk factor & $\mathrm{n}$-tot & n-pu & n-tot & $n-p u$ & $\mathrm{n}$-tot & n-pu & $n$-to & ll n-pu \\
\hline Immobility & 13 & 1 & 63 & 39 & 77 & 13 & 20 & 7 \\
\hline Inactive & 16 & 1 & 67 & 39 & 113 & 16 & 24 & 8 \\
\hline Limited (or no reaction) & 14 & 1 & 83 & 43 & 90 & 14 & 19 & 6 \\
\hline Vasoactive medication & 10 & 1 & 53 & 29 & 76 & 10 & 10 & 4 \\
\hline Incontinence & 12 & 1 & 73 & 42 & 94 & 15 & 6 & 2 \\
\hline Oedema & 8 & 1 & 1 & 1 & 27 & 7 & 12 & 7 \\
\hline Surgery <24 hours & 7 & 1 & 20 & 9 & 32 & 0 & 5 & 2 \\
\hline Surgery $>24$ hours & 17 & 1 & 55 & 28 & 73 & 16 & 14 & 5 \\
\hline
\end{tabular}

\section{DISCUSSION}

The number of units that participated in the study in each country varied significantly. An explanation for this variance might be that some units that were approached decided not to participate because of the additional work involved in collecting the data or because they did not want to make their prevalence data public. Whatever the reason, the only way to improve our standard of care in this area is to share our prevalence and incidence data in relation to the pressure ulcer prevention and treatment protocols we use. The importance of this is seen in a number of articles that discuss different methods of how to improve the care of patients at risk for pressure ulcer development (Sacharok and Drew, 1998; Cockbill-Black et al., 1999; Logan et al., 1999; Weststrate and Bruining, 1996).

\section{Need for standardisation}

The variety of risk assessment scales implies that there is no general consensus among the intensive care nursing organisations on which risk assessment scale to use for intensive care patients. Barrett (1990) suggests using the Waterlow scale for the assessment of risk of pressure ulcer development in intensive care patients. So far only Weststrate et al. (1998) has evaluated the Waterlow scale for this patient group. Other investigators have found various cut-off levels when they validated the Braden pressure ulcer risk assessment scale for intensive care patients (Jiricka et al., 1995; Fife et al., 2001). Braden and Bergstrom suggest that these differences can often be related to the influence of external factors, such as staffing ratios (Braden and Bergstrom, 1996). However, regardless of which risk assessment scale is chosen, the scale needs to be validated based on the patients and working conditions of the ICU in which it is being used. Besides validation, standardisation in using a particular pressure ulcer risk assessment scale for ICU patients would make comparison and analysis possible between the different pressure ulcer prevention programmes used on different ICUs.

Table 6. Number of patients (with or without a pressure ulcer) using each mattress type at the time of assessment, and the number of patients ( $\mathrm{n}$ ) with a pressure ulcer using each type of mattress

$\begin{array}{lllll}\text { Type of mattress } & \text { Denmark } & \text { Germany } & \text { Italy } & \text { Netherlands } \\ \text { Hospital mattress } & 6 & 39(14) & 31(1) & 3 \\ \text { Foam mattress } & 3 & 22(13) & 6(1) & - \\ \text { Air mattress } & - & - & 5(2) & 7(4) \\ \text { Water mattress } & 8 & - & 23(1) & - \\ \text { Alternating mattress } & - & 10(5) & 14(2) & 4 \\ \text { Low-air-loss mattress } & - & 24(16) & 44(9) & 10(5) \\ \text { Rotation bed } & - & - & 11(5) & 2(1) \\ \text { Other } & 7(1) & 4(1) & 16 & - \\ \text { Total no. of patients } & 24(1) & 99(49) & 150(21) & 26(10)\end{array}$




\section{Frequency of risk assessment}

The frequency of assessment depends on the nature of patients. The more rapidly changes are likely to occur in a patient's condition, the more frequently the risk assessment should be carried out. For ICUs, it has been recommended that risk assessment should be carried out once every 24 hours (Braden and Bergstrom, 1996). This is endorsed by the fact that the majority of patients, regardless of whether they already had a pressure ulcer, could be classified as being at high risk of developing one (Table 4). Despite this finding, $34 \%$ of the units only assessed their patients' risk once per week. This is far too long, as nursing awareness lags behind the actual status of most patients, and in some cases, pressure ulcers can develop in only a few hours.

It is striking that there was a large amount of missing data concerning the calculated risk levels for Germany and Italy. It is possible that the risk assessment scale used for these patients did not discriminate between different risk levels.

\section{Risk factors}

This study found that immobility, inactivity, impaired sensitivity, vasoactive medication and incontinence were the most important risk factors for the development of a pressure ulcer. Impaired sensitivity was also found by Carlson et al. (1999). Other studies have found these risk factors to be important in pressure ulcer development, including inactivity and the use of vasoactive medication by Batson et al., (1993), immobility by Jesurum et al. (1996), and incontinence by Allman et al. (1995).

\section{Prevalence rates}

The pressure ulcer prevalence rates (Stage II or higher) for the various countries varied significantly. The most extreme figure was a prevalence of $49 \%$ on the critical care units in Germany. In an earlier study, O'Dea (1995) had reported that Germany had a prevalence of $4 \%$ of pressure ulcers among hospital patients, but this study was performed on general patients and not only on intensive care patients.

No other study so far has compared the pressure ulcer prevalence on ICUs in Europe. Prevalence studies are difficult to compare with incidence studies because they include every patient with a new pressure ulcer within a specific time frame (Lake, 1999). Prevalence studies take a 'snapshot' at one moment in time. Another reason why comparison is difficult is that some studies include stage I (non-blanching erythema) as being a pressure ulcer, although this stage is reversible. In our study, we excluded this stage because it has been shown that nurses do not always know the difference between blanching and non-blanching erythema. At pressure ulcer stages II, III and IV, the skin is broken and so is easier than stage I to classify accurately (Collier, 1999).

\section{Type of support mattress}

The use of support mattresses is interesting. Italy used the largest number of types of mattresses and Germany the smallest. Besides the low-air-loss and alternating mattress, the water mattress is frequently used. Compared with a standard mattress, Cullum et al. (2000) found that the water mattress prevented the development of a pressure ulcer more effectively, but he found no significant difference between a water mattress and an alternating mattress (Cullum et al., 2000). Furthermore, there was no evidence that low-air-loss mattresses are more effective at preventing pressure ulcers than alternating mattresses, although the study suggested limited evidence for a reduced incidence of pressure ulcers in ICU patients with low-air-loss mattresses (Cullum et al., 2000). In order to decide which kind of technology (lowair-loss or alternating) is more effective in prevention of pressure ulcers in intensive care patients, ICUs should standardise the type of used mattresses to one of these two alternatives.

Italy and the Netherlands were the only countries that used rotation beds. Primarily, rotation beds have been used in (ventilated) patients to prevent further pulmonary complications (Nelson and Choi, 1992). However, rotation beds have a secondary effect in preventing the development of a pressure ulcer. The original, primary reason for using these expensive beds was not given in this study.

The study found that Germany had the highest proportion of at-risk patients (high and medium). The finding that caregivers waited too long before installing a special mattress for high and medium risk patients may be the reason why Germany had the highest prevalence of pressure ulcers in this study. As the average 'incubation period' of a pressure ulcer is 4 days (Kosiak, 1959), it is critical that some sort of pressure relief is installed as soon as a patient has an increased risk (medium or high) of developing pressure ulcers. Compared to other countries, Germany had the lowest proportion of high-risk patients using a special mattress.

\section{CONCLUSIONS}

It is evident that the prevalence of pressure ulcers in intensive care patients varies between the different European countries, and different units use different protocols in relation to risk assessment scales and type of mattresses. Protocols give professional guidance regarding procedures to follow when a pressure ulcer is likely to occur in a patient. Apparently some protocols are more successful than others in the prevention of pressure ulcers. In order to prevent the development of pressure ulcers in intensive care patients in Europe, it is essential that protocols for prevention of pressure ulcers are standardised for ICU patients.

The EPUAP can play an important role in developing such a protocol because they have an extensive network of clinical healthcare professionals. For successful implementation of such a protocol, collaboration with other European professional societies such as the European federation of Critical Care Nursing associations (EfCCNa) and the European Society of Intensive Care Medicine (ESICM) is critical. Together with monthly prevalence studies at each unit, successful strategies can be identified and further implemented in all ICUs in Europe.

\section{ACKNOWLEDGEMENTS}

I would like to thank KCI Medical, a medical supply company specialising in intensive care equipment, including support mattresses, for their help. The national KCI Medical offices of Denmark, Germany, Italy and the Netherlands distributed and collected the questionnaires and entered the gathered data into a database. However, the study results have been reported by the author independently of KCI Medical and no financial support has been received in preparing this report. I would also like to thank Mrs Rachel Stewart for her help in preparing this article.

\section{REFERENCES}

Allman RM, Goode PS, Patrick MM, Burst N, Bartolucci AA. (1995). Pressure ulcer risk factors among hospitalized patients with activity limitation [see comments]. Journal of American Medical Association; 273: 865-870.

Barrett E. (1990). Pressure sores in intensive care. Intensive Therapy and Clinical Monitoring; September/October: 158-167.

Batson S, Adam S, Hall G, Quirke S. (1993). The development of a pressure area scoring system for critically ill patients: a pilot study. Intensive and Critical Care Nursing; 9: 146-151.

Bours GJJW, De Laat E, Halfens RJG, Lubbers M. (2001). Prevalence, risk factors and prevention of pressure ulcers in Dutch intensive care units. Intensive Care Medicine (in press). 
Braden BJ, Bergstrom N. (1996). Risk assessment and risk-based programs of prevention in various settings. Ostomy/Wound Management; 42(Suppl): 6S-12S.

Carlson EV, Kemp MG, Shott S. (1999). Predicting the risk of pressure ulcers in critically ill patients. American Journal of Critical Care; 8: 262-269.

Cockbill-Black S, Bond J, Bersee-Mills A, Warren K, Hammerton S, Found D, Daley L. (1999). Audit of pressure area care and documentation. Professional Nurse; 15: 173-176.

Collier, M. (1999). Blanching and non-blanching hyperaemia. J Wound Care; 8: 63-64.

Cullum N, Deeks J, Sheldon T, Song F, Fletcher A. (2000). Beds, mattresses and cushions for pressure sore prevention and treatment. The Cochrane Library; 4: 1-33.

Dealey C. (1991). The size of the pressure-sore problem in a teaching hospital. Journal of Advanced Nursing; 16: 663-670.

Fife C, Otto G, Capsuto EG, Brandt K, Lyssy K et al. (2001). Incidence of pressure ulcers in a neurologic intensive care unit. Critical Care Medicine; 29: 283-290.

Haalboom JR. (1992). De kosten van decubitus. [Cost aspects of decubitus.] Nederlands Tijdschrift voor Geneeskunde; 135: 606-610.

Inman KJ, Sibbald WJ, Rutledge FS, Clark BJ. (1993). Clinical utility and costeffectiveness of an air suspension bed in the prevention of pressure ulcers [see comments]. Journal of American Medical Association; 269: 1139-1143.

Jackson C. (1999). The revised Jackson/Cubbin Pressure Area Risk Calculator. Intensive and Critical Care Nursing; 15: 169-175.

Jesurum J, Joseph K, Davis JM, Suki R. (1996). Balloons, beds, and breakdown. Effects of low-air loss therapy on the development of pressure ulcers in cardiovascular surgical patients with intra-aortic balloon pump support. Critical Care Nursing Clinics in North America; 8: 423-440.

Jiricka MK, Ryan P, Carvalho MA, Bukvich J. (1995). Pressure ulcer risk factors in an ICU population. American Journal of Critical Care; 4: 361-367.
Kemp MG, Keithley JK, Smith DW, Morreale B. (1990). Factors that contribute to pressure sores in surgical patients. Research in Nursing and Health; 13: 293-301.

Kosiak M. (1959). Etiology and pathology of ischemic ulcers. Archives of Physical Medicine and Rehabilitation; 40: 62-69.

Lake NO. (1999). Measuring incidence and prevalence of pressure ulcers for intergroup comparison. Advanced Wound Care; 12: 31-34.

Logan J, Harrison MB, Graham ID, Dunn K, Bissonnette J. (1999). Evidence-based pressure-ulcer practice: the Ottawa model of research use. Canadian Journal of Nursing Research; 31: 37-52.

Nelson LD, Choi SC. (1992). Kinetic therapy in critically ill trauma patients. Clinics in Intensive Care; 3: 248-252.

O'Dea K. (1995). The prevalence of pressure sores in four European countries. Journal of Wound Care; 4: 192-195.

Sacharok C, Drew J. (1998). Use of a total quality management model to reduce pressure ulcer prevalence in the acute care setting. Journal of Wound Ostomy and Continence Nursing; 25: 88-92.

Waterlow JA. (1995). Reliability of the Waterlow score [letter; comment]. Journal of Wound Care; 4: 474-475.

Weststrate JT, Bruining HA. (1996). Pressure sores in an intensive care unit and related variables: a descriptive study. Intensive and Critical Care Nursing; 12: 280-284.

Weststrate JT, Hop WC, Aalbers AG, Vreeling AW, Bruining HA. (1998). The clinical relevance of the Waterlow pressure sore risk scale in the ICU. Intensive Care Medicine; 24: 815-820.

Xakellis GC, Frantz RA. (1996). The cost-effectiveness of interventions for preventing pressure ulcers. Journal of American Board of Family Practitioners; 9: 79-85. 